\title{
Comparison of the clinical outcomes between antiviral-naïve patients treated with entecavir and lamivudine-resistant patients receiving adefovir add-on lamivudine combination treatment
}

\author{
Hong Joo Kim, Soo Kyung Park, Hyo Joon Yang, Yoon Suk Jung, Jung Ho Park, Dong Il Park, Yong Kyun Cho, \\ Chong Il Sohn, Woo Kyu Jeon, Byung Ik Kim, and Kyu Yong Choi \\ Division of Gastroenterology, Department of Internal Medicine, Kangbuk Samsung Hospital, Sungkyunkwan University School of \\ Medicine, Seoul, Korea
}

Background/Aims: To analyze the effects of preexisting lamivudine (LAM) resistance and applying antiviral treatment (adefovir [ADV] add-on LAM combination treatment) on long-term treatment outcomes, and comparing the clinical outcomes of antiviral-naïve chronic hepatitis B patients receiving entecavir (ETV) monotherapy.

Methods: This study enrolled 73 antiviral-naïve patients who received 0.5 -mg ETV as an initial therapy and 54 patients who received ADV add-on LAM combination treatment as a rescue therapy from July 2006 to July 2010.

Results: During 24-month treatments, the decreases in serum log10HBV-DNA values (copies $/ \mathrm{mL}$ ) were significantly greater in the antiviral-naïve patients treated with ETV than the patients receiving ADV add-on LAM combination treatment. The biochemical response rates for alanine aminotransferase normalization at 6 months (ETV) and 12 months (ADV add-on LAM) were $90.4 \%(66 / 73)$ and $77.8 \%(42 / 54)$, respectively ( $P=0.048)$. A Kaplan-Meier analysis indicated that the rates of serologic response, viral breakthrough, and emergence of genotypic resistance did not differ significantly between the two patient groups. There were also no significant intergroup differences in the rates of disease progression (PD) and new development of hepatocellular carcinoma (HCC).

Conclusions: The long-term clinical outcomes of antiviral-naïve patients treated with ETV and LAM-resistant patients receiving ADV add-on LAM combination treatment were comparable in terms of the emergence of HCC and disease progression. (Clin Mol Hepatol 2016;22:350-358)

Keywords: Entecavir; Adefovir; Chronic hepatitis B; Disease progression; Cirrhosis

\section{INTRODUCTION}

Chronic hepatitis B (CHB) is a major health problem, with an es- timated prevalence of 350 million carriers worldwide. Each year CHB is responsible for more than 1 million deaths from cirrhosis and hepatocullar carcinoma (HCC). ${ }^{1}$ The risk of disease progres-

\footnotetext{
Abbreviations:

ADV, adefovir; AFP, alpha fetoprotein; ALT, alanine aminotransferase; AST, aspartate aminotransferase; CHB, chronic hepatitis B; CTP, Child-Turcotte-Pugh; DNA, deoxyribonucleic acid; ETV, entecavir; HCC, hepatocellular carcinoma; LAM, lamivudine; LdT, telbivudine; NA, nucleos(t)ide analogue; PCR, polymerase chain reaction; $\mathrm{PD}$, disease progression; $\mathrm{SD}$, standard deviation; TFV, tenofovir; $Y M D D$, mutations in the tyrosine-methionine-aspartate-aspartate
}

\section{Corresponding author : Hong Joo Kim}

Department of Internal Medicine, Kangbuk Samsung Hospital, Sungkyunkwan University School of Medicine, 29 Saemunan-ro, Jongnogu, Seoul 03181, Korea

Tel: +82-2-2001-8330, Fax: +82-2-2001-8340

E-mail: hongjoo3.kim@samsung.com 
Hong Joo Kim, et al.

Outcomes of ETV and combination therapies

sion (PD) to cirrhosis and HCC, and liver-related mortality are strongly correlated with serum HBV-DNA levels, and the suppression of HBV-DNA to undetectable levels has been adopted as an important endpoint for antiviral treatment in patients with $\mathrm{CHB} .{ }^{2-6}$ Evidence-based medicine has demonstrated that effective antiviral treatment of $\mathrm{CHB}$ reduces the risk of long-term complications such as the emergence of liver cirrhosis and HCC, and improves patient survival. ${ }^{7,8}$ Currently available antiviral drugs for CHB include peginterferon- $\alpha$ and nucleos(t)ide analogue (NA) HBV polymerase inhibitors [lamivudine(LAM), adefovir (ADV), entecavir (ETV), telbivudine (LdT) and tenofovir (TFV)].

ETV is a potent antiviral agent with a high genetic barrier and it induces a significant decline in viral loads in both HBeAg-positive and HBeAg-negative treatment-naïve patients. ${ }^{9,10}$ Genotypic resistance to ETV in treatment-naïve patients is rare, occurring in $1.2 \%$ of patients after 5 years of therapy, while only $0.8 \%$ of patients develop a viral breakthrough due to ETV resistance." ETV monotherapy as a rescue therapy for CHB patients with LAM resistance resulted in continued viral suppression and biochemical and serologic responses; however, sequential ETV monotherapy resulted in a 5-year cumulative probability of genotypic ETV resistance of $51 \%$. " As a rescue therapy for CHB patients with LAM resistance, $A D V$ add-on LAM combination treatment is superior to sequential ADV monotherapy, resulting in effective viral suppression and a reduced risk of developing genotypic resistance. ${ }^{12,13}$

Long-term viral suppression by drugs with potent antiviral activity and a low rate of drug resistance to achieve a durable response could be a common principle in the prevention of deterioration of liver function (to hepatic decompensation), reduction or prevention of progression to liver cirrhosis (and its complications) and/or HCC, and the prolongation of survival. ${ }^{14,15}$ Consensus has been reached that treatment must be administered long-term due to the high rate of virologic relapse when nucleos(t)ide analogue (NA) therapy is discontinued. So far, the effect of preexisting LAM resistance and adopted antiviral treatment (ADV add-on LAM combination treatment) on the long-term treatment outcomes, such as the deterioration of liver function, progression to liver cirrhosis (and its complications) and/or HCC remains unclear. The aim of the current study is to analyze the effect of preexisting LAM resistance and adopted antiviral treatment (ADV add-on LAM combination treatment) on the long-term treatment outcomes, comparing the clinical outcomes of antiviral-naïve CHB patients with patients on ETV monotherapy.

\section{MATERIALS AND METHODS}

\section{Study population}

Two hundred twenty-eight patients who underwent antiviral treatment for $\mathrm{CHB}$, including ETV $0.5 \mathrm{mg}$ once a day as an initial therapy and ADV add-on LAM combination treatment as a rescue therapy for pre-existing genotypic resistance to LAM from July 2006 to July 2010, were considered to be eligible candidate for the current study. Among them, 101 patients were excluded from the final analyses due to the following reasons: (1) initially diagnosed HCC $(n=3)$, (2) adoption of ETV $1.0 \mathrm{mg}$ once a day as a rescue treatment for genotypic resistance to LAM $(n=23)$, (3) drug noncompliance $(n=27)$, (4) co-infection with hepatitis $C$ virus $(n=2)$, and (5) inadequate follow-up (less than 12 months) or follow-up loss $(n=46)$. Ultimately, 73 antiviral naïve patients who were initiated on ETV $0.5 \mathrm{mg}$ once a day as an initial therapy and 54 patients on ADV add-on LAM combination treatment as a rescue therapy for preexisting genotypic resistance to LAM were enrolled in the current retrospective cohort study. The median (range) duration of follow-up for these patients was 50 (23 83) months. This study was conducted in accordance with the principles of the Declaration of Helsinki. Our study protocol obtained the approval of the Ethics Committee of Kangbuk Samsung Hospital (KBC11054, approved at 2011-05-16).

\section{Efficacy end points}

The primary end point was the time to disease progression (PD), defined by the following criteria: (1) escalation of the Child-Turcotte-Pugh (CTP) score by more than 2 points, (2) new appearance of complications of liver cirrhosis such as ascites, bleeding gastroesophageal varices, and hepatic encephalopathy, and (3) new development of hepatocellular carcinoma and/or cirrhosis. The secondary end points included the following: (1) reduction in the serum HBV-DNA, defined as a mean $\log _{10}$ HBV-DNA (copies/ $\mathrm{mL}$ ) decrease, from the baseline to a designated time point after the initiation of the antiviral treatment, (2) the proportion of patients with undetectable HBV-DNA ( $<116$ copies/mL by quantitative PCR assay) 6 months and 12 months after the initiation of ETV $0.5 \mathrm{mg}$ once a day and ADV add-on LAM combination treatment, respectively, (3) the proportion of patients with alanine aminotransferase (ALT) normalization 6 months and 12 months after the initiation of ETV $0.5 \mathrm{mg}$ once a day and ADV add-on LAM combination treatment, respectively, (4) the proportion of 
patients with HBeAg negative conversion and/or seroconversion, and (5) the proportion of patients with the emergence of viral breakthrough and genotypic resistance.

\section{Laboratory assays}

Routine liver biochemical tests including serum ALT, aspartate aminotransferase (AST), albumin, total bilirubin, prothrombin time, and creatinine level were determined at the baseline and every 3 months during the treatment with ETV $0.5 \mathrm{mg}$ once a day or ADV add-on LAM combination treatment, using a sequential multiple autoanalyzer (Hitachi, Tokyo, Japan). HBV-DNA was measured at the baseline and every 3 months during the treatment with ETV $0.5 \mathrm{mg}$ once a day or ADV add-on LAM combination treatment by the real-time PCR method using a COBAS TaqMan analyser (Roche Diagnostics, Mannheim, Germany) with a lower limit of detection of 116 copies $/ \mathrm{mL}$. Hepatitis B e antigen (HBeAg) and antibody against $\mathrm{HBeAg}$ were tested with commercial radioimmunoassay kits (Abbott Laboratory, North Chicago, IL, USA). Mutations in the tyrosine-methionine-aspartate-aspartate (YMDD) motif of the HBV-DNA polymerase gene (rt204 and rt180) were identified at the baseline using restriction fragment mass polymorphism (RFMP) analysis, as previously described. ${ }^{16}$ For the detection of ADV resistant mutation, RFMP analysis was performed at the baseline and at the time of the virologic breakthrough, as previously described. ${ }^{16}$ The genotytpic analysis obtained by RFMP was confirmed using sequencing analysis. For detection of ETV resistance, direct sequencing using a Dye Primer Cycle Sequencing Kit and the Open Gene Automated DNA Sequencing System (Visible Genetics Inc., Toronto, Canada) was used at the baseline and at the time of the virologic breakthrough.

\section{Follow-up and definitions}

Patients were monitored at the baseline and every 3 months thereafter during the dosing period. Patients underwent clinical assessment of tolerability and drug compliance (checked with a pill count), physical examination, and blood drawing for measurements of laboratory parameters and HBV-DNA levels. The upper limit of normal for ALT was $40 \mathrm{IU} / \mathrm{L}$. A complete responder was considered as a patient who had undetectable serum HBV-DNA by PCR ( $<116$ copies $/ \mathrm{mL}$ ) 6 and 12 months after the initiation of ETV $0.5 \mathrm{mg}$ once a day and ADV add-on LAM combination therapy, respectively. A nonresponder (initial treatment failure) was considered as a patient with a decline in the HBV-DNA level to less than $2 \log _{10}$ copies/m value 6 and 12 months after the initiation of ETV $0.5 \mathrm{mg}$ once a day and ADV add-on LAM combination therapy, respectively. A virologic breakthrough was considered as an increase in the HBV-DNA level to more than $1 \log _{10}$ value compared with the trough level after an initial period of complete or partial response to that treatment. A biochemical breakthrough was defined as an increase in ALT activity above the ULN after an initial return to normal levels.

\section{Statistical analyses}

For a comparison of the antiviral efficacy, and the frequency of the occurrence of viral breakthrough and genotypic resistance between each treatment group during the study period, an independent samples $t$-test for continuous data and Fisher's exact test for categorical data were carried out. The efficacy parameters included serum HBV-DNA and ALT levels. Cumulative HBeAg loss/seroconversion was compared between each treatment group using a

Table 1. Baseline demographics and clinical characteristics of patients in the ETV group and the ADV add-on LAM combination treatment group.

\begin{tabular}{|c|c|c|c|}
\hline Characteristics & ETV group $(n=73)$ & ADV add-on LAM group $(n=54)$ & $\boldsymbol{P}$-value \\
\hline Age (mean $\pm S D$, years) & $47.2 \pm 11.1$ & $47.7 \pm 10.5$ & 0.812 \\
\hline Male gender (\%) & $45(61.6)$ & $36(66.7)$ & 0.581 \\
\hline Follow-up period (mean $\pm S D$, months) & $43.8 \pm 11.9$ & $60.0 \pm 15.6$ & $<0.01$ \\
\hline Liver cirrhosis (\%) & $21(28.8)$ & $13(24.1)$ & 0.686 \\
\hline CTP class (A/B/C, \%) & $15(71.4) / 6(28.6) / 0(0)$ & $12(85.8) / 1(7.1) / 1(7.1)$ & 0.605 \\
\hline positive HBeAg (\%) & $42(57.5)$ & $38(70.4)$ & 0.193 \\
\hline HBV genotype C (\%) & $73(100)$ & $54(100)$ & 1.0 \\
\hline Baseline ALT (mean \pm SD, IU/L) & $219.2 \pm 269.2$ & $162.0 \pm 205.3$ & 0.142 \\
\hline Baseline $\log _{10}$ HBV-DNA (mean \pm SD, copies/mL) & $6.73 \pm 1.88$ & $6.44 \pm 2.02$ & 0.825 \\
\hline
\end{tabular}

ETV, entecavir; ADV, adefovir; LAM, lamivudine; SD, standard deviation; CTP, Child-Turcotte-Pugh; HBeAg, hepatitis B e antigen; HBV, hepatitis B virus; ALT, alanine aminotransferase. 
log rank test with Kaplan Meier analysis. Statistical analysis was conducted using the PASW statistics package 18 (IBM, Armonk, New York, USA). P-values less than 0.05 were considered significant. Statistical review of this study was performed by a biomedical statistician (Seungho Ryu) affiliated to our institution.

\section{RESULTS}

\section{Comparison of baseline characteristics between the patients group with ETV monotherapy and ADV add- on LAM combination treatment}

The baseline demographic and disease characteristics of the enrolled antiviral-naïve patients with ETV $0.5 \mathrm{mg}$ treatment and patients with ADV add-on LAM combination treatment as a rescue therapy for preexisting genotypic resistance to LAM were well balanced except for the follow-up period (Table 1). All patients

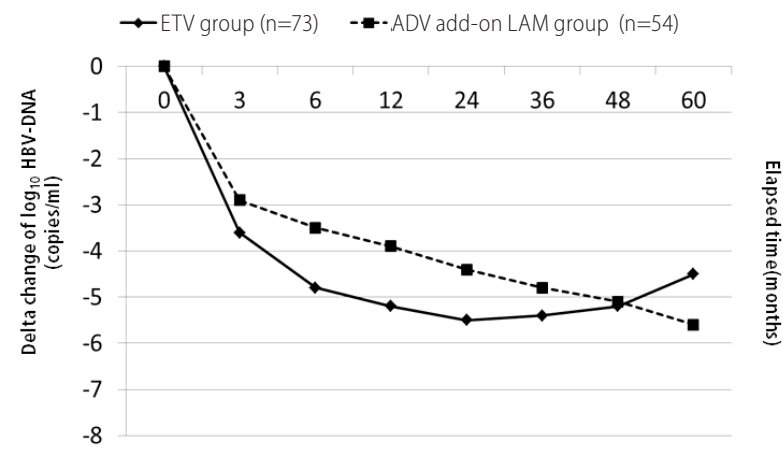

Figure 1. Comparisons of the reduction of serum $\log _{10} \mathrm{HBV}$-DNA values (copies/mL) from baseline to 3, 6, 12, 24, 36, 48, and 60 months between antiviral-naïve patients receiving initial ETV treatment and LAM-resistant patients receiving ADV add-on LAM combination treatment.

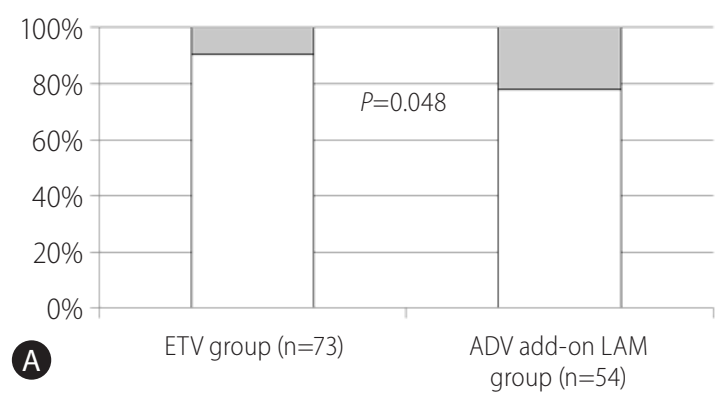

had genotype C HBV infection. The proportion of patients with liver cirrhosis, CTP class, HBeAg positivity, baseline ALT level and baseline $\log _{10} \mathrm{HBV}$-DNA (copies $/ \mathrm{mL}$ ) values were comparable between the two patient groups. The mean \pm standard deviation (SD) duration of LAM treatment before adoption of ADV add-on LAM combination treatment was $13.9 \pm 20.2$ months, and the composition of baseline genotypic resistance profile for these patients was as follows; rtM204I only - 15 (27.8\%), rtM204I + rtL180M - 13 (24.1\%), rtM204V + rtL180M - 19 (35.2\%), and rt$\mathrm{M} 204 \mathrm{I} / \mathrm{V}+\mathrm{rtL} 180 \mathrm{M}-7$ (13.0\%).

\section{Comparison of virologic, serologic, biochemical responses and genotypic resistance between the patients group with ETV monotherapy and ADV add-on LAM combination treatment}

The mean \pm SD reduction of the serum $\log _{10} H B V$-DNA (copies/ $\mathrm{mL}$ ) from the baseline to $3,6,12,24,36,48$ and 60 months was $-3.60 \pm 1.71,-4.80 \pm 1.60,-5.20 \pm 1.66,-5.52 \pm 1.95,-5.39 \pm$ $2.09,-5.15 \pm 2.26$, and $-4.45 \pm 2.03$ in the ETV group, and -2.86 $\pm 1.36,-3.46 \pm 1.54,-3.88 \pm 1.79,-4.38 \pm 2.13,-4.82 \pm 1.96$ $-5.10 \pm 2.06$, and $-5.60 \pm 1.82$ in the ADV add-on LAM group, respectively (Fig. 1). For comparisons of repetitive serum $\log _{10} \mathrm{H}$ BV-DNA (copies $/ \mathrm{mL}$ ) values between the two treatment group, we adopted linear mixed model and found that more significant decrease in serum $\log _{10} \mathrm{HBV}$-DNA (copies $/ \mathrm{mL}$ ) values was noted in ETV treatment group compared to ADV add-on LAM combination group (coefficient 0.2319261, standard error 0.3009238, z 0.77, $P>|z| 0.441,95 \%$ confidence interval $-0.3578737 \sim 0.8217258$ ).

After 6 months of ETV treatment and 12 months after ADV addon LAM treatment, ALT normalization was observed in $90.4 \%$ $(66 / 73)$, and $77.8 \%(42 / 54)$ of patients who received ETV and

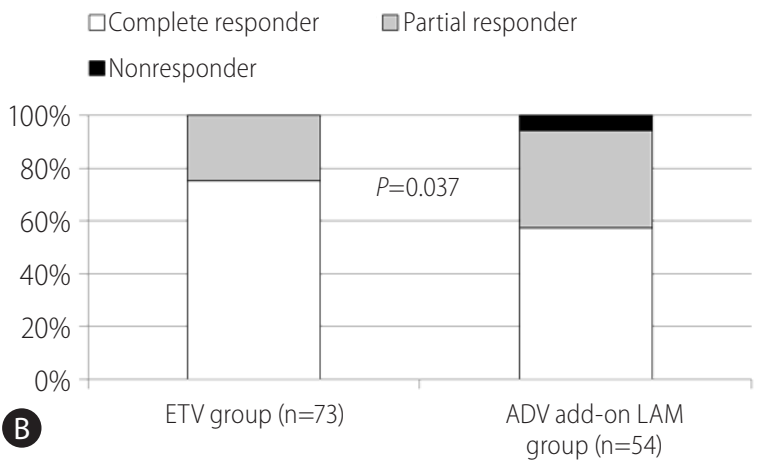

Figure 2. Comparisons of (A) biochemical responses and (B) virologic responses between initial ETV treatment (6 months after the baseline) and rescue ADV add-on LAM combination treatment (12 months after the baseline). ALT, alanine aminotransferase; ULN, upper limit of normal; ETV, entecavir; ADV, adefovir; LAM, lamivudine. 
ADV add-on LAM treatment, respectively (Fig. $2 A, P=0.048$ by Chi-square test). The rates of HBV-DNA PCR undetectability $(<116$ copies $/ \mathrm{mL}$ ) 6 and 12 months after the initiation of ETV and ADV add-on LAM combination treatment were $75.3 \%$ (55/73) and $57.4 \%$ (31/54), respectively (Fig. 2B, $P=0.037$ ).

The cumulative $\mathrm{HBeAg}$ loss and seroconversion rates were not significantly different between the ETV and ADV add-on LAM treatment group by Kaplan-Meier analysis with log rank comparison (Fig. $3 A)$. The cumulative rates of the emergence of viral breakthrough and genotypic resistance were also not significantly different between the ETV and ADV add-on LAM treatment groups by KaplanMeier analysis with log rank comparison (Fig. 3B).
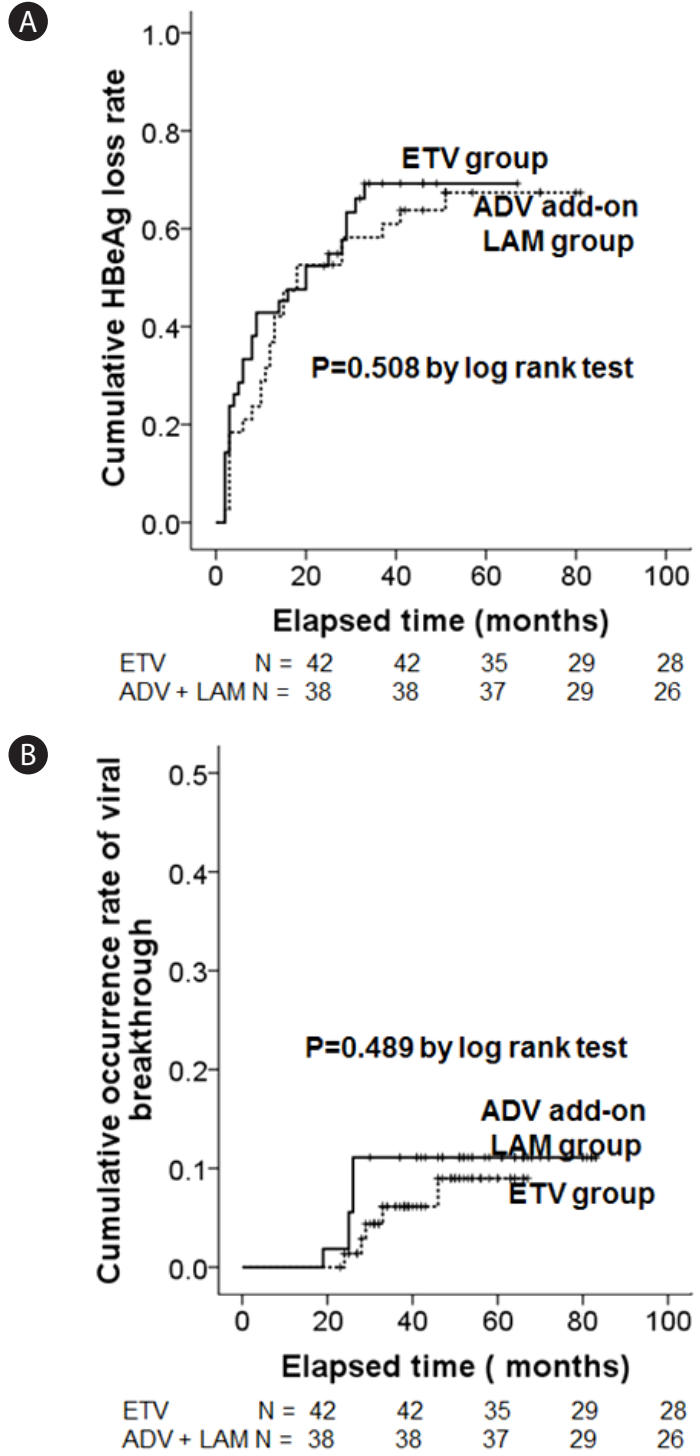

\section{Disease progression and HCC development}

For the median (duration) follow-up of 50 (23-83) months, PD was observed in seven patients (9.6\%) in the ETV group and eight patients (14.8\%) in the ADV add-on LAM group. Among the seven PD patients in the ETV group, five patients developed new HCC lesions, one patient developed new liver cirrhosis, and the remaining one patient died from liver failure with hepatorenal syndrome. Among the eight PD patients in the ADV add-on LAM group, five patients developed new liver cirrhosis, two patients developed new HCC lesions, and the remaining one patient became

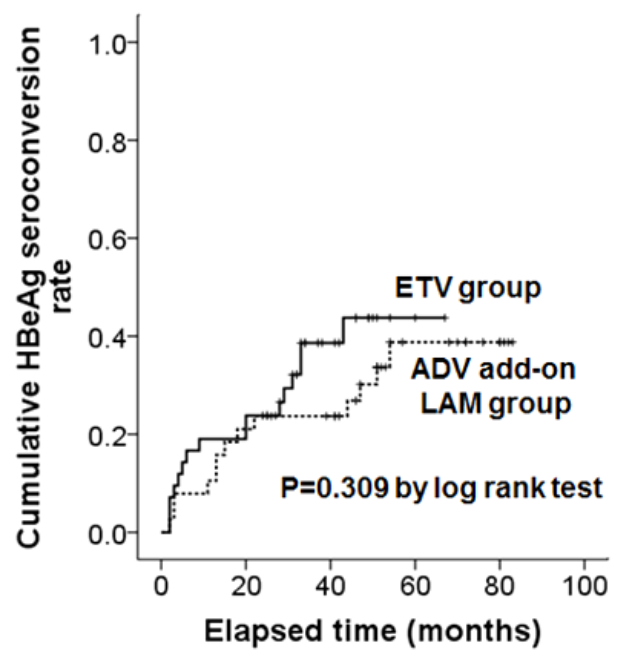

$\begin{array}{lrllll}\text { ETV N } & =42 & 42 & 35 & 29 & 28 \\ \text { ADV + LAM N }=38 & 38 & 37 & 29 & 26\end{array}$

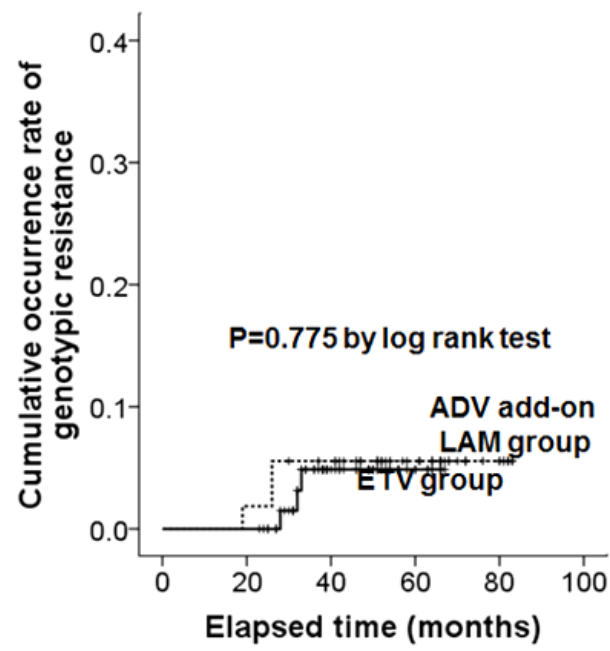

$\begin{array}{lrllll}\text { ETV N }=42 & 42 & 35 & 29 & 28\end{array}$

Figure 3. Comparisons of (A) cumulative HBeAg loss and HBeAg seroconversion rates and (B) cumulative occurrence rates of viral breakthrough and genotypic resistance between initial ETV treatment and rescue ADV add-on LAM combination treatment. ETV, entecavir; ADV, adefovir; LAM, lamivudine. 

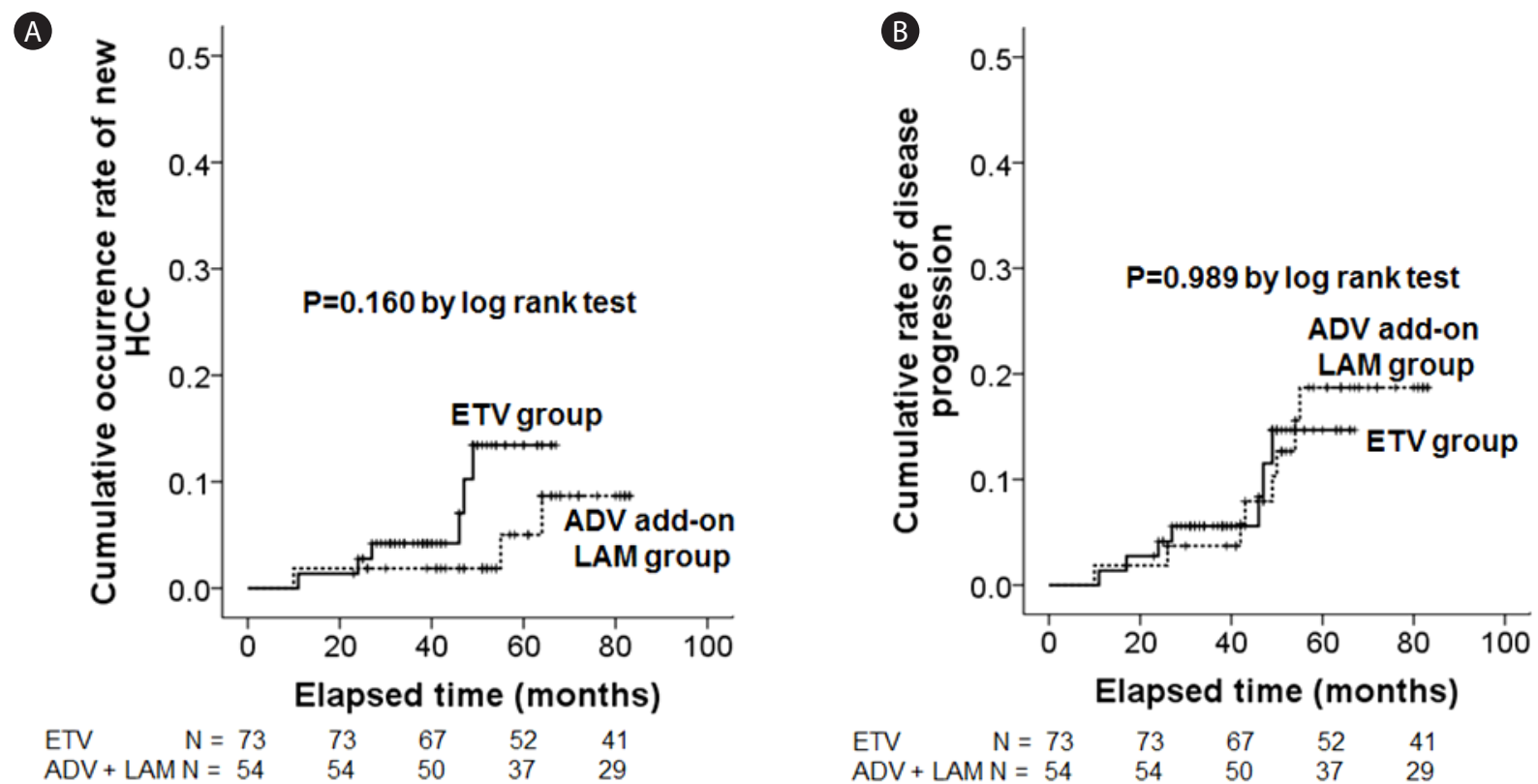

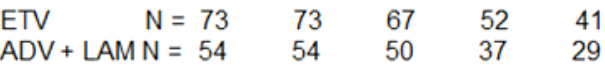

Figure 4. Comparisons of cumulative occurrence rates of new HCC and disease progression. HCC, hepatocellular carcinoma; ETV, entecavir; ADV, adefovir; LAM, lamivudine.

Table 2. Comparison of results from mixed-effects logistic regression analyses of the cumulative occurrence rate of new HCC and PD between the ETV group and the ADV add-on LAM combination treatment group

\begin{tabular}{lccccc}
\hline Characteristics & Odds ratio & Standard error & $\mathbf{z}$ & $\mathbf{P > | z |}$ & $\mathbf{9 5 \% ~ C l}$ \\
\hline $\begin{array}{l}\text { Comparison of the occurrence rate of new HCC } \\
\text { between the two treatment group }\end{array}$ & 2.076757 & 1.224772 & 1.24 & 0.215 & $0.6537134 \sim 6.597568$ \\
$\begin{array}{l}\text { Comparison of the occurrence rate of PD between the } \\
\text { two treatment group }\end{array}$ & 0.5795236 & 0.4631516 & -0.68 & 0.495 & $0.1210031 \sim 2.77553$ \\
\hline
\end{tabular}

HCC, hepatocelllular carcinoma; PD, disease progression; ETV, entecavir; ADV, adefovir; LAM, lamivudine; Cl, confidence interval.

deceased by liver failure with hepatic encephalopathy and sepsis. By Kaplan-Meier analysis with log rank comparison, there were no significant differences in the cumulative rates of PD and new HCC development between the ETV group and ADV add-on LAM treatment group, respectively (Fig. 4). For control of bias caused by the large number of exclusion during the patients enrollment $(n=101)$, we performed mixed-effects logistic regression analyses, and found that there were no significant differences in terms of odds ratio in the cumulative occurrence rate of new HCC and PD between ETV group and ADV add-on LAM treatment group, respectively (Table 2).

\section{DISCUSSION}

This study provides the first comparison of the treatment outcomes, including the cumulative occurrence rate of PD, the new development of HCC and antiviral efficacies of ETV $0.5 \mathrm{mg}$ in treatment-naïve patients and ADV add-on LAM combination treatment in LAM-resistant patients with CHB. As previously reported, ${ }^{7}$ long-term nucleos(t)ide analogue therapy for adults with CHB prevents or delays the development of long-term complications including decompensated cirrhosis, CHB-related HCC or death in patients with CHB. In the current study, we defined the $P D$, a direct clinical comparator of the clinical outcome of different nucleos(t)ide analogue therapies for adults with $\mathrm{CHB}$, as the following: (1) escalation of the Child-Turcotte-Pugh (CTP) score by more than 2 points, (2) new appearance of complications of liver cirrhosis such as ascites, bleeding gastroesophageal varices, and hepatic encephalopathy, and (3) new development of hepatocellular carcinoma and/or cirrhosis. Interestingly, there were no significant differences in the cumulative rates of PD and new HCC development between the ETV and ADV add-on LAM treatment groups, respectively (Fig. 4). A previous multicenter, randomized, 
double-blind, placebo-controlled prospective study ${ }^{17}$ showed that patients with the genotypic YMDD mutation to LAM were more likely to have an increased Child-Pugh score than those without YMDD mutations. In addition, a meta-analysis performed by Zhang et al., ${ }^{7}$ in which the long-term complications were defined as the end events of the clinic including (1) decompensated cirrhosis which included the occurrence of ascites, esophageal/gastric variceal bleeding, spontaneous bacterial peritonitis or hepatic encephalopathy, (2) HBV-related HCC diagnosed histologically or by diagnostic imaging methods, given an alpha fetoprotein (AFP) level above $400 \mathrm{ng} / \mathrm{mL}$, or (3) HBV-related death, showed that patients with antiviral drug resistance had a higher probability of long-term complications than patients without drug resistance (relative risk 2.64). However, in the current study, there were no significant differences in the cumulative rates of PD (the definition of PD of the current study was almost identical to that of the meta-analysis performed by Zhang et al. ${ }^{7}$ ) between antiviral-naïve patients with ETV $0.5 \mathrm{mg}$ medication and patients with ADV addon LAM combination treatment as a rescue therapy for proven genotypic resistance to LAM. We speculated that additional factor(s) such as the effectiveness of rescue antiviral treatment (ADV add-on LAM combination treatment in the current study) in patients with genotypic resistance to LAM could account for this difference in outcomes among the current study and previous ones. Actually, the major event-free survival was significantly better in patients remaining in virologic remission (maintenance of the initial virologic response without development of a virologic breakthrough) than in those with a virologic breakthrough, or no response to LAM. ${ }^{18}$ Eun et al. ${ }^{19}$ also showed that HCC developed less frequently in $\mathrm{CHB}$ patients with compensated liver cirrhosis who were in sustained viral suppression (HBV-DNA $<141,500$ copies $/ \mathrm{mL}$ ) than those with a viral breakthrough or a suboptimal response (HBV-DNA $\geq 141,500$ copies $/ \mathrm{mL}$ ). The above-mentioned findings show that persistent viral suppression demonstrated by a persistently low serum HBV-DNA level could be a determining factor of the long-term clinical outcomes in CHB patients with nucleos(t)ide analogue therapy. In the current study, the decreases in serum $\log _{10} \mathrm{HBV}$-DNA (copies $/ \mathrm{mL}$ ) were significantly more conspicuous in antiviral naïve patients with ETV treatment than patients with ADV add-on LAM combination treatment, until 24 months after the initiation of each antiviral treatment (Fig. 1). However, there were no significant differences in the reduction of serum $\log _{10}$ HBV-DNA (copies $/ \mathrm{mL}$ ) between the two treatment groups 36,48 , and 60 months after the initiation of ETV or ADV add-on LAM combination treatment. Though, it seemed that the short-term antiviral potency of ETV was significantly higher than ADV add-on LAM combination treatment, the long-term antiviral potency was similar between the two treatments, and this analogy for long-term suppression of HBV-DNA between ETV and ADV add-on LAM treatment could explain the results of the current study which showed similar long-term clinical outcomes (cumulative occurrence rate of PD) between the two treatment groups.

Genotypic resistance to ETV in treatment-naïve patients, occurring in $1.2 \%$ of patients after 5 years of therapy, is rarely reported. ${ }^{11}$ In the current study, the cumulative occurrence rate of viral breakthrough and genotypic resistance to ETV in antiviral-naïve patients with CHB was $9.0 \%$ (standard error, SE 4.0\%) and 4.9\% (SE 2.7\%) during a median (range) follow-up of 42 (24-67) months, respectively. There were no significant differences compared to the cumulative occurrence rate of viral breakthrough $(11.1 \%$, SE $4.3 \%)$ and genotypic resistance $(5.6 \%$, SE $3.1 \%)$ to ADV add-on LAM combination treatment. The above-mentioned occurrence rates of viral breakthrough and genotypic resistance of ETV were relatively higher than previously reported. "We speculated that although, we had strictly enrolled antiviral-naïve patients for the initial ETV $0.5 \mathrm{mg}$ treatment, a small proportion of the CHB patients with previous exposure to LAM could have been enrolled in the current study population for the ETV group. In Korea, LAM has been prescribed for many years in various clinical settings, including private clinics by non-experts in liver diseases, and patients might not be aware that they have received LAM, but only thought they had prescriptions of 'liver protective medications'. It was impossible to completely sort out the patients with previous LAM exposure by history-taking only at the initial visit. Another possible explanation for the relatively high occurrence rate of viral breakthrough and genotypic resistance to ETV may be the genotypic composition of the HBV infection enrolled in the current study. The genotype of HBV enrolled in the current study was $\mathrm{C}$ in $100 \%$ of the patients. It was reported that CHB patients with HBV genotype $C$ had a higher viral load than genotype $B$, and had a higher risk of developing HCC and showing disease progression due to the wild-type precore 1896 sequence, and basal core promoter (BCP) A1762T/G1764A mutation. ${ }^{20}$ However, a recent meta-analysis consistently found no significant association between HBV genotype and response to nucleos(t)ide analogues. ${ }^{21}$

Because of the limitation inherent in our study design (retrospective cohort study), head-to-head comparisons of each clinical variables for each enrolled patient of the two study groups could not be done in the current study. However, we attempted to con- 
trol the selection bias inherent in the retrospective study design by roughly matching the baseline clinic-epidemiologic characteristics between the two study groups of the current study (Table 1). Additionally, more than 6 months of follow-up loss before recognition of viral breakthrough and commitment of genotypic mutation test was noted in 21 patients out of 54 ADV add-on LAM group (38.9\%) and this high proportion of patients with longterm follow-up loss may be a causative factor for the relatively high baseline $\log _{10}$ HBV-DNA and serum ALT level in patients with ADV add-on LAM combination treatment in the current study.

In conclusion, the current study suggests that there were no significant differences in the long-term clinical outcomes, including the emergence of HCC and disease progression, between antiviral-naïve patients with initial ETV treatment and patients with ADV add-on LAM combination treatment as a rescue therapy for genotypic resistance to lamivudine.

\section{Authors' contributions}

HJ Kim had the original idea for the study, and shared responsibility for data collection, data processing, statistical analyses, writing, and reviewing the manuscript. SK Park, HJ Yang, YS Jung, JH Park, DI Park, YK Cho, CI Sohn, WK Jeon, BI Kim and KY Choi reviewed the manuscript and gave their critical revisions.

\section{Conflicts of Interest}

The authors have no conflicts to disclose.

\section{REFERENCES}

1. Lee WM. Hepatitis B virus infection. N Engl J Med 1997;337:17331745.

2. Iloeje UH, Yang HI, Su J, Jen CL, You SL, Chen CJ. Risk Evaluation of Viral Load Elevation and Associated Liver Disease/Cancer-In HBV (the REVEAL-HBV) Study Group. Predicting cirrhosis risk based on the level of circulating hepatitis B viral load. Gastroenterology 2006;130:678-686.

3. Chen CJ, Yang HI, Su J, Jen CL, You SL, Lu SN, et al. Risk of hepatocellular carcinoma across a biological gradient of serum hepatitis $B$ virus DNA level. JAMA 2006;295:65-73.

4. Liaw YF, Kao JH, Piratvisuth T, Chan HL, Chien RN, Liu CJ, et al. Asian-Pacific consensus statement on the management of chronic hepatitis B: a 2012 update. Hepatol Int 2012;6:531-561.

5. Lok AS, McMahon BJ. Chronic hepatitis B: update 2009. Hepatology 2009;50:661-662.

6. European Association For The Study Of The Liver. EASL Clinical Prac- tice Guidelines: Management of chronic hepatitis B virus infection. J Hepatol 2012;57:167-185.

7. Zhang QQ, An X, Liu YH, Li SY, Zhong Q, Wang J, et al. Long-Term Nucleos(t)ide Analogues Therapy for Adults With Chronic Hepatitis $B$ reduces the Risk of Long-Term Complications: a meta-analysis. Virol J 2011;8:72

8. Yang YF, Zhao W, Zhong YD, Xia HM, Shen L, Zhang N. Interferon therapy in chronic hepatitis $B$ reduces progression to cirrhosis and hepatocellular carcinoma: a meta-analysis. J Viral Hepat 2009 ;16:265-271.

9. Lai CL, Shouval D, Lok AS, Chang TT, Cheinquer H, Goodman Z, et al. Entecavir versus lamivudine for patients with $\mathrm{HBeAg}$-negative chronic hepatitis B. N Engl J Med 2006;354:1011-1020.

10. Gish RG, Lok AS, Chang TT, de Man RA, Gadano A, Sollano J, et al. Entecavir therapy for up to 96 weeks in patients with $\mathrm{HBeAg}$ positive chronic hepatitis B. Gastroenterology 2007;133:1437-1444.

11. Tenney DJ, Rose RE, Baldick CJ, Pokornowski KA, Eggers BJ, Fang $J$, et al. Long-term monitoring shows hepatitis $B$ virus resistance to entecavir in nucleoside-naive patients is rare through 5 years of therapy. Hepatology 2009;49:1503-1514.

12. Gaia S, Barbon V, Smedile A, Olivero A, Carenzi S, Lagget M, et al. Lamivudine-resistant chronic hepatitis $B$ : an observational study on adefovir in monotherapy or in combination with lamivudine. J Hepatol 2008;48:540-547.

13. Chen EQ, Wang LC, Lei J, Xu L, Tang H. Meta-analysis: adefovir dipivoxil in combination with lamivudine in patients with lamivudineresistant hepatitis B virus. Virol J 2009;6:163.

14. European Association for the Study of the Liver. EASL Clinical Practice Guidelines: management of cholestatic liver diseases. J Hepatol 2009;51:237-267.

15. Liaw YF, Leung N, Guan R, Lau GK, Merican I. Asian-Pacific consensus statement on the management of Chronic hepatitis $B$ : an update. J Gastroenterol Hepatol 2003;18:239-245.

16. Kim HS, Han KH, Ahn SH, Kim EO, Chang HY, Moon MS, et al. Evaluation of methods for monitoring drug resistance in chronic hepatitis $B$ patients during lamivudine therapy based on mass spectrometry and reverse hybridization. Antivir Ther 2005;10:441-449.

17. Liaw YF, Sung JJ, Chow WC, Farrell G, Lee CZ, Yuen H, et al. Lamivudine for patients with chronic hepatitis $B$ and advanced liver disease. N Engl J Med 2004;351:1521-1531.

18. Papatheodoridis GV, Dimou E, Dimakopoulos K, Manolakopoulos S, Rapti I, Kitis G, et al. Outcome of hepatitis B e antigen-negative chronic hepatitis $B$ on long-term nucleos(t)ide analog therapy starting with lamivudine. Hepatology 2005;42:121-129.

19. Eun JR, Lee HJ, Kim TN, Lee KS. Risk assessment for the development of hepatocellular carcinoma: according to on-treatment viral response during long-term lamivudine therapy in hepatitis B virusrelated liver disease. J Hepatol 2010;53:118-125. 
20. Yu MW, Yeh SH, Chen PJ, Liaw YF, Lin CL, Liu CJ, et al. Hepatitis B virus genotype and DNA level and hepatocellular carcinoma: a prospective study in men. J Natl Cancer Inst 2005;97:265-272.

21. Wiegand J, Hasenclever D, Tillmann HL. Should treatment of hepa- titis B depend on hepatitis B virus genotypes? A hypothesis generated from an explorative analysis of published evidence. Antivir Ther 2008;13:211-220. 